Case Report

\title{
Magnetic resonance imaging of pilocytic astrocytomas in adults with histopathologic correlation: a report of six consecutive cases
}

\author{
Alberto Di Napoli ${ }^{1}$, Paolo Spina ${ }^{2}$, Alessandro Cianfoni ${ }^{1,3}$, Luca Mazzucchelli ${ }^{2}$, Emanuele Pravatà $1,3, *$ (D) \\ ${ }^{1}$ Department of Neuroradiology, Neurocenter of Southern Switzerland, 6903 Lugano, Switzerland \\ ${ }^{2}$ Cantonal Institute of Pathology, 6601 Locarno, Switzerland \\ ${ }^{3}$ Faculty of Biomedical Sciences, Università della Svizzera Italiana, 6900 Lugano, Switzerland \\ *Correspondence: emanuele.pravata@eoc.ch (Emanuele Pravatà)
}

DOI:10.31083/j.jin2004105

This is an open access article under the CC BY 4.0 license (https://creativecommons.org/licenses/by/4.0/).

Submitted: 24 March 2021 Revised: 13 April 2021 Accepted: 10 May 2021 Published: 30 December 2021

Pilocytic astrocytoma is a WHO grade I tumor usually diagnosed in pediatric patients, and rarely encountered in the adult population. Therefore, available information about the magnetic resonance imaging characteristics of adult pilocytic astrocytoma is scarce. We report on the MRI features and corresponding histopathologic findings of six consecutive aPA cases diagnosed. The tumors were encountered in both infra- and supratentorial compartments, and their MRI characteristics were quite heterogeneous. Features included the typical solid-cystic appearance located in the cerebellum as well as the relatively unusual multifocal and/or hemorrhagic features located intra-ventricularly. The aPA MRI characteristics are remarkably variable, and might mimic those of higher grade tumors in adult patients.

Keywords

Pilocytic astrocytoma; Adult; MRI; Brain tumor; Aqueduct; Hydrocephalus; Multifocal

\section{Introduction}

Pilocytic astrocytoma (PA) accounts for approximately $1.5 \%$ of adult brain tumors, with only sporadic cases being reported after the age of $50[1,2]$. While the imaging features of pediatric PA (pPA) have been extensively reported in the literature [3], descriptions of adult PA (aPA) magnetic resonance imaging (MRI) characteristics are scarce. To our knowledge, only a few descriptions are available from case reports focusing on clinical presentations and/or surgical findings [4-6]. Here, we report on the MRI signal, morphological appearance, contrast enhancement patterns, and locations of six sporadic (i.e., NF1-unrelated) aPA cases, which were consecutively imaged, biopsied and/or treated at our institution, and provide histopathologic correlation.

\section{Cases presentation}

We report on the cases of five males and one female, over 18 years of age at diagnosis, who were clinically evaluated, imaged, surgically treated and/or biopsied at the Neurocenter of Southern Switzerland. In all cases, multiplanar T2-weighted turbo-spin-echo (TSE), T2*-weighted gradient-echo (GRE), fluid-attenuated-inversion-recovery T2-weighted, diffusion-weighted-imaging, and pre- and post-gadolinium T1-weighted fast-GRE images, were available. High-resolution steady-state free-precession (SSFP) (cases 1 and 4 only), and T2* perfusion-weighted imaging (case 4 only), were also obtained as specified.

Brain tissue specimens were routinely prepared in formalin-fixed paraffin-embedded (FFPE) blocks before H\&E staining. Immuno-histo-chemistry (IHC) was performed on 2- $\mu$ m-thick FFPE sections. Antibodies for ATRX (polyclonal; 1:400; Sigma Aldrich; St. Louis, Missouri/USA), CD3, CD20, CD34, CD68, GFAP, IDH1, Ki67, NFP and p53 were tested. Exon 4 of the IDH1 gene, exon 4 of the $I D H 2$ gene and exon 15 of the $B R A F$ gene were tested by direct sequencing, without finding any mutation. All tumors were pathologically confirmed, according to the 2016 WHO CNS Tumor Classification [7]. Histopathology descriptions of the $\mathrm{H} \& \mathrm{E}$ stains are listed for each case in the figure legends.

\subsection{Case 1}

A 71-year-old man presented with a 2-year history of urinary incontinence, ataxia, gait disturbances and episodic memory loss. MRI imaging demonstrated a $4 \mathrm{~mm}$, welldefined, nodular enhancing lesion within the Sylvian aqueduct, causing obstructive hydrocephalus (Fig. 1). A biopsy was obtained and a third-ventriculostomy was performed, leading to clinical improvement. A one-year follow-up MRI demonstrated stability of the residual lesion (not shown).

\subsection{Case 2}

A 43-year-old man complained of 2-year history of gait disturbances and fatigue. On MRI (Fig. 2) a multicystic expansile lesion centered in the left cerebellar hemisphere was detected, with well-defined margins and intralesional hemorrhage. A solid mural nodule showed partial but intense contrast-enhancement. There was moderate compression of the fourth ventricle, without hydrocephalus. After total resection, the patient's symptoms gradually resolved. 

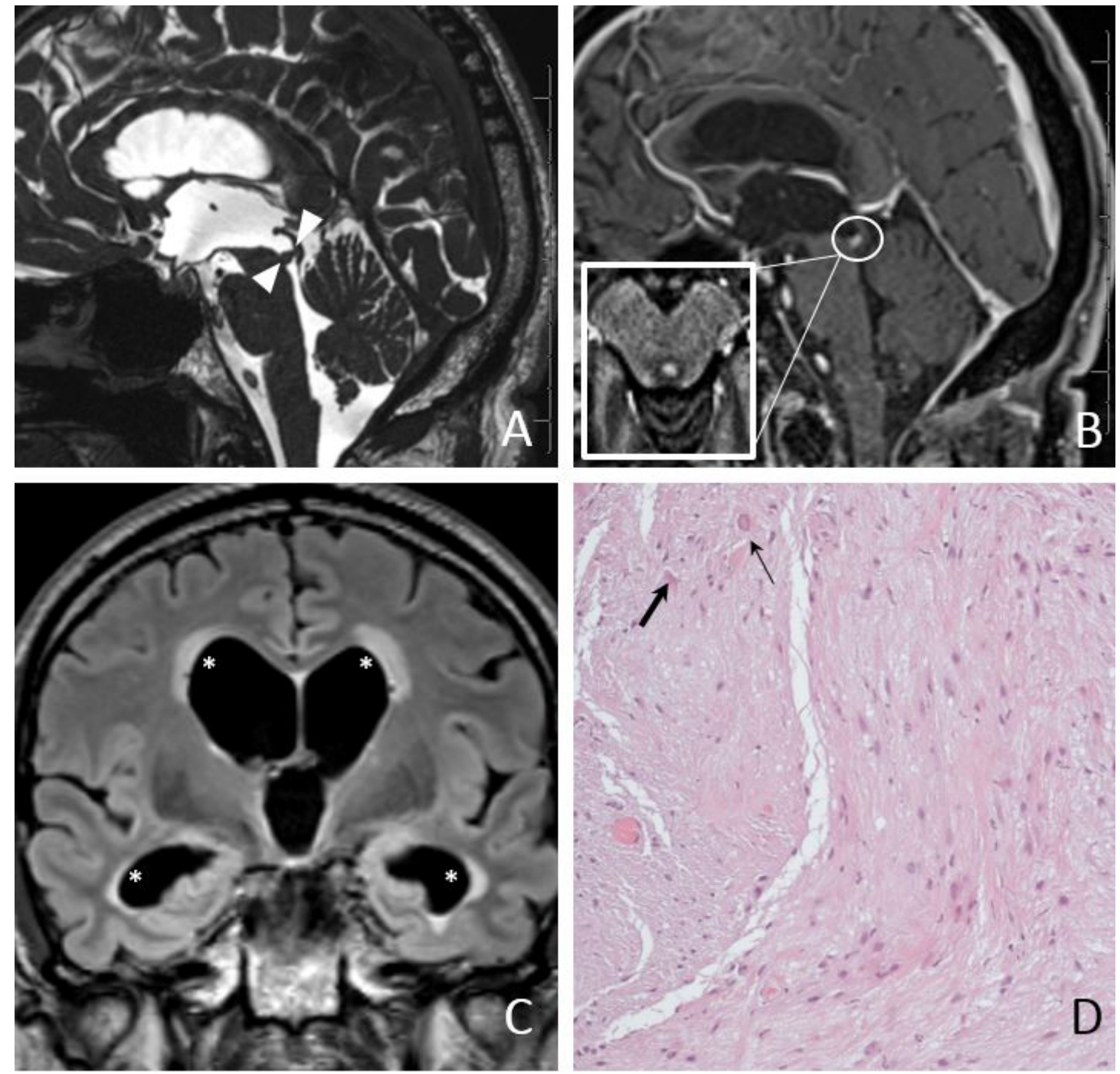

Fig. 1. Case 1. Unusual presentation of an intra-aqueductal aPA causing obstructive hydrocephalus. Sagittal plane reconstructed 3D steady-state (A) and postgadolinium 3D T1-weighted fast-gradient-echo (B) images, show a small, oval-shaped, enhancing nodule within the mesencephalic aqueduct (arrowheads). Coronal T2-FLAIR (C) demonstrates associated triventricular hydrocephalus (asterisks) with transependymal CSF resorption. Microscopically (D) this tumor exhibits a typical densely fibrillary matrix, comprising spindle cells with elongated nuclei and mild atypia, Rosenthal fibers (thick arrow) and eosinophilic granular bodies (thin arrow).

\subsection{Case 3}

A 31-year-old female presented with a 2-year history of worsening migraine, and mild gait disturbances. MRI (Fig. 3) revealed a solid exophytic mass originating from the posterior aspect of the medulla oblongata. The lesion presented mildly hyperintense T2-signal, and minimally increased water diffusivity, with multinodular/patchy enhancement. No mass effect was present. At a two-year follow-up, MRI showed tumor growth, which prompted complete surgical resection with resulting resolution of symptoms.

\subsection{Case 4}

A 21-year-old man presented with a 3-month history of vertigo and bilateral visual acuity deficit, and bilateral papillary edema. MRI (Fig. 4) showed a heterogeneous multicystic mass with a solid component, originating from the quadrigeminal plate and extending into the fourth ventricle, causing hydrocephalus. The solid part exhibited intense contrast-enhancement. Dynamic susceptibility perfusion imaging showed increased relative cerebral blood volume (rCBV) values (1.79) with respect to the adjacent unaffected brainstem. After subtotal resection and ventricular shunt, patient's symptoms improved. Residual tumor was stable at one-year MRI follow-up (not shown).

\subsection{Case 5}

A 19-year-old man presented with a first time tonic-clonic seizure, following alcohol and cannabis abuse. A multifocal presentation was seen on MRI (Fig. 5) with two left temporal lobe nodular lesions. Lesion 1 was centered in the cortico-subcortical junction, had a target appearance with a T2-hypointense peripheral rim exhibiting intense contrast- 

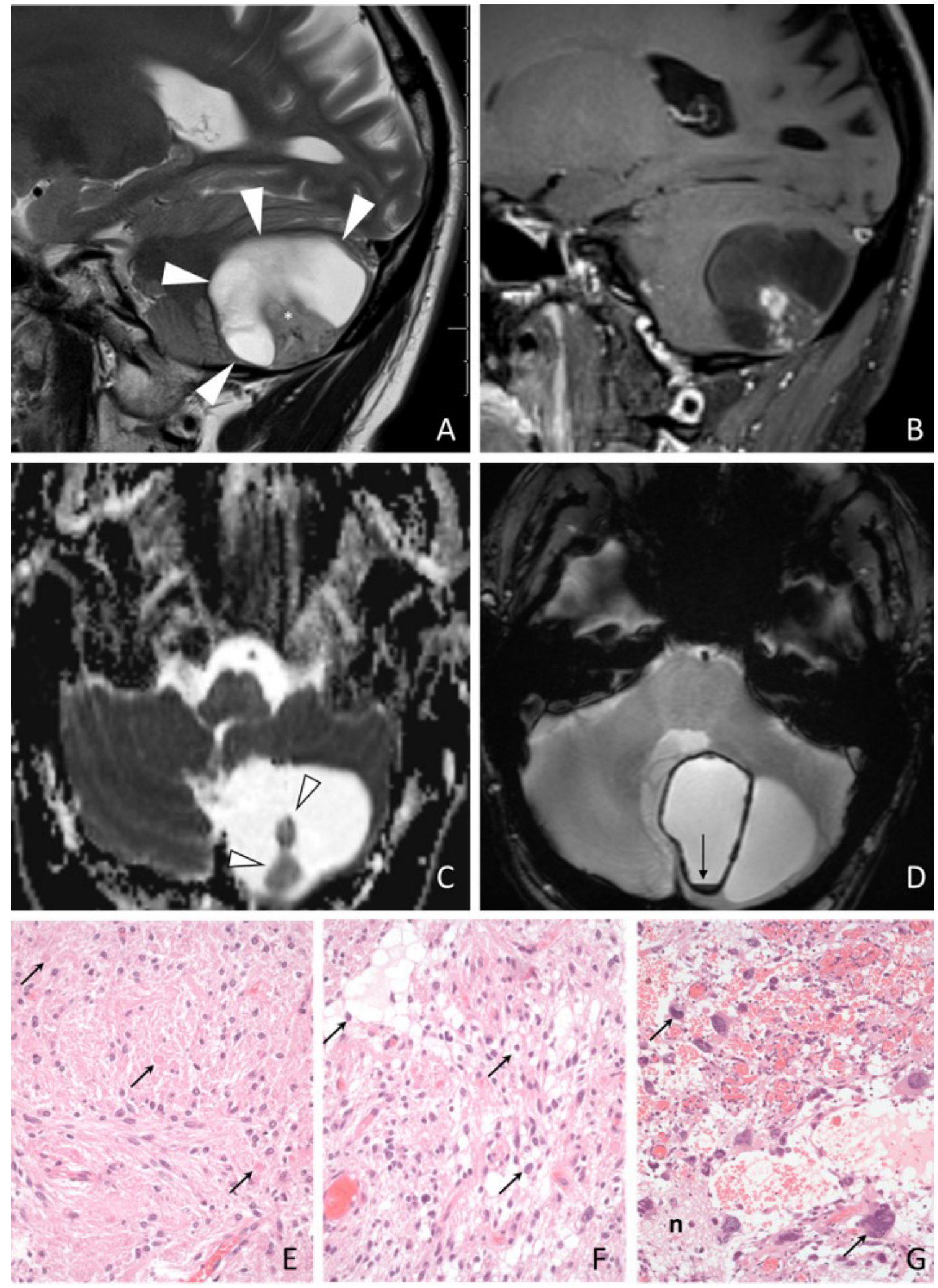

Fig. 2. Case 2. Cystic-Hemorrhagic aPA. Sagittal T2-weighted (A) and T1-weighted images after contrast (B) show a T2-bright cystic lesion (arrowheads), with a slightly hyperintense nodule with patchy enhancement located in the cerebellar hemisphere. ADC signal intensity (C) is slightly increased in comparison to the adjacent white matter (arrowheads). Axial T2*-weighted images (D) demonstrate signs of hemosiderin deposition lining one of the cyst walls, and a small fluid level, indicating chronic intratumoral hemorrhage. Histologically, the solid part of the tumor corresponds to areas with a piloid pattern, with cytologically bland cells and eosinophilic granular bodies (arrows in E), as well as other areas (F) with a looser architecture exhibiting microcystic changes, round-to-ovoid cells (arrows), a pseudoangiomatous hypervascular pattern and large pleomorphic cells (arrows in G). These were interpreted as regressive changes, because of the presence of only rare mitoses and the very low Ki-67 proliferative index (about 3\%). 

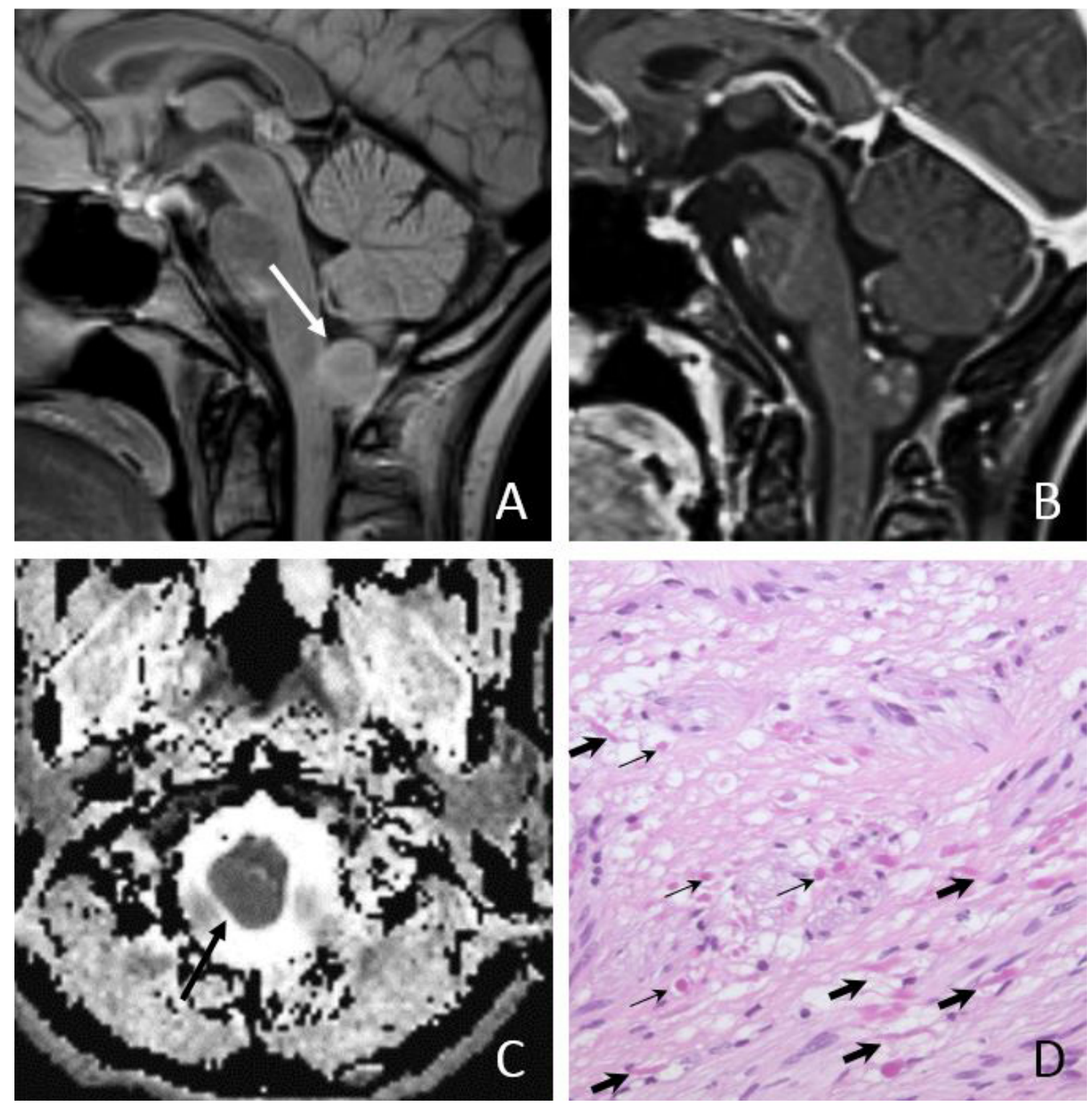

Fig. 3. Case 3. Exophytic aPA. Sagittal plane reconstructed 3D T2-FLAIR (A) shows a solid, exophytic mass originating from the dorsal aspect of the medulla and growing into the cisterna magna (arrow). The mass presents substantial T2-FLAIR signal isointensity compared to the cerebellar cortex, and a patchy enhancement after gadolinium injection (B). On the ADC map (C) the lesion showed minimally increased diffusivity (arrow), as compared to the medulla. Microscopically (D) this tumor exhibits a loose, fibrillary matrix rich in Rosenthal fibers and eosinophilic granular bodies (thin arrows), comprising elongated, spindle cells, without relevant atypia (thick arrows).

enhancement, and was surrounded by vasogenic edema. Lesion 2 was a subependymal nodule abutting into the ventricular trigone, with heterogeneous T2-signal and central contrast-enhancement. Lesion 1 was biopsied, which revealed aPA. The patient declined surgery and was lost to follow-up.

\subsection{Case 6}

A 38-year-old man presented with a 4-week history of phosphenes, ocular pain and vomiting. MRI (Fig. 6) revealed a heterogeneous solid intraventricular mass in the left frontal horn compressing the foramen of Monro, with multiple foci of internal chronic hemorrhage, heterogeneous inter- nal patchy enhancement with multiple cystic and/or necrotic components, as well as increased water diffusivity with respect to the unaffected parenchyma. The mass caused ventricular entrapment. The patient underwent complete resection and his symptoms resolved.

\section{Case discussion}

In the pediatric population PA exhibits a wide, yet well described range of imaging features. While the most typical imaging pattern is that of an oval mass with cystic and enhancing solid components in the cerebellar hemisphere or anterior visual pathways, more heterogeneous appearances are also relatively frequent $[3,8]$. In our aPA cases we en- 

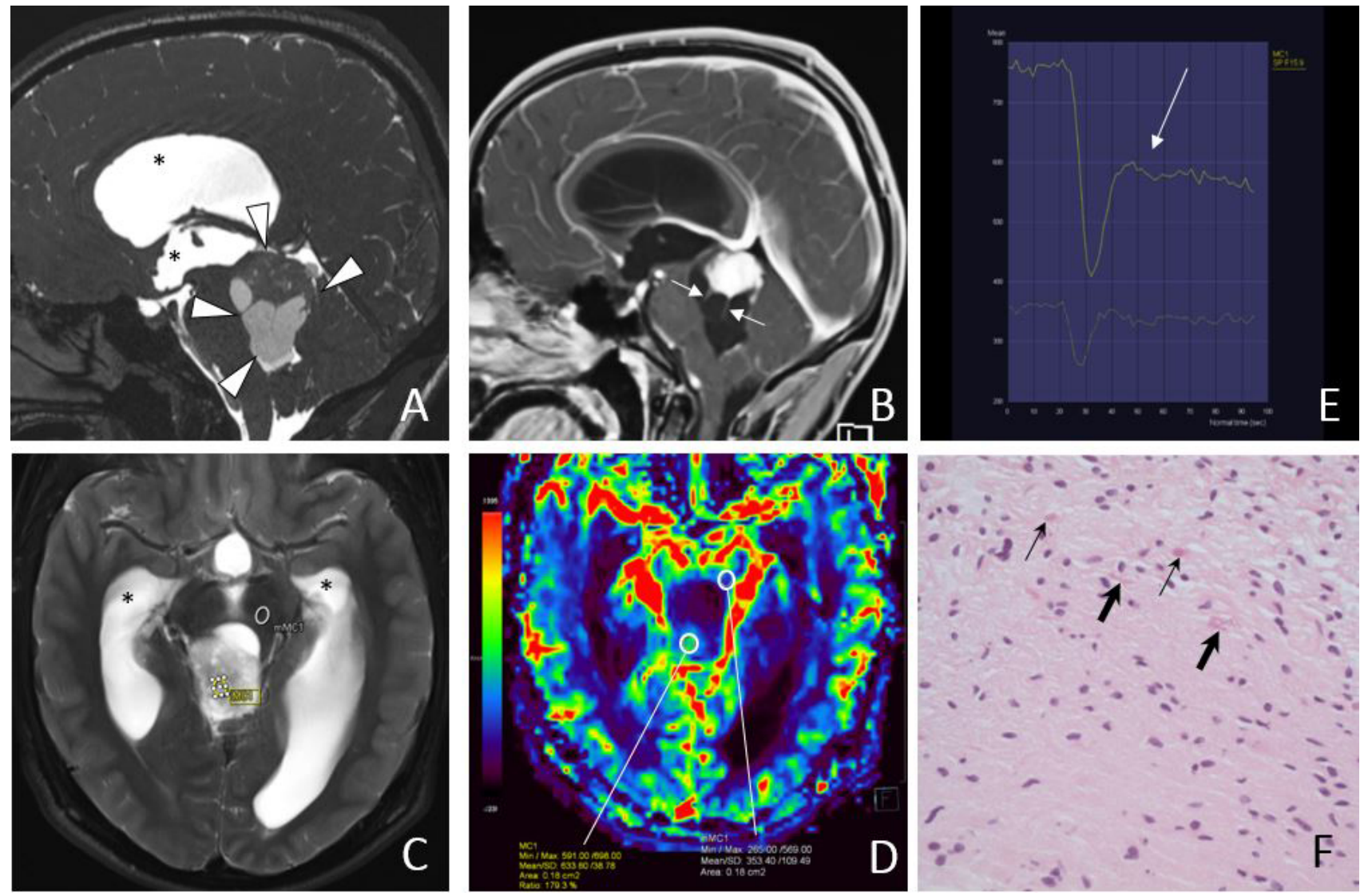

Fig. 4. Case 4. aPA causing obstructive hydrocephalus. Sagittal plane reconstructed 3D steady-state "CISS" (A) and post-gadolinium 3D T1 fast GRE (B) images demonstrate a solid-cystic mass (arrowheads in A) with intense contrast enhancement (arrows in B), originating from the quadrigeminal plate, and causing aqueduct compression with triventricular hydrocephalus. The solid component of the mass shows inhomogeneous T2 hyperintensity (C - axial T2 TSE image), and moderately increased rCBV (1.79) as compared to the normal WM (ROIs D - see also ROIs in C). The corresponding perfusion time-signal curve (E, arrow) shows only partial signal recovery after first pass contrast enhancement, consistent with a fenestrated brain-tumor-barrier (please see discussion section for more details). The histological findings (F) demonstrate a densely, fibrillary texture with bland cells, some Rosenthal fibers (thick arrows) and eosinophilic granular bodies (thin arrows).

countered a variety of MRI features, with an assortment of associated heterogeneous histopathology patterns, on which we comment below.

\subsection{Morphology and MRI signal features}

We found the classical pPA appearance of a large cyst with enhancing mural nodule in only two out of six adult cases. In the remaining four cases we observed a nodular pattern with a completely solid or mixed cystic-solid appearance. The T2 signal intensity was variable, including frankly heterogeneous, high signal textures (in cases $4,5,6$ ), as well as a relatively homogenous and slightly hyperintense signal, compared to the cortex (in cases 1, 2, 3).

Interestingly, despite being low-grade tumors, enhancement often occurs in PA, which is thought to occur due to fenestrations in the blood-tumor barrier leading to the contrast extravasation [9]. Enhancement patterns were patchy/irregular in 50\% (cases 2, 6, 3), nodular/solid in two patients (cases 1, 4, 5-lesion 1), and rim-like in one patient (case 5-lesion 2). Hemorrhage and necrosis are uncommon findings in pPAs (about 8\%) [3]. However, two (28.6\%) adult patients (2 and 6) remarkably showed signs of intratumoral hemorrhage on $\mathrm{T} 2 *$ images. The variegated enhancement patterns and the presence of hemorrhage may mimic more common lesions in the adults, such as metastasis, hemangioblastoma, or higher grade glioma [10]. On the other hand, some aPA features, including cystic changes, well defined margins and relatively small size, were also described in IDH-mutant gliomas [11, 12]. In our aPA cases, water diffusivity was always increased and/or similar to the unaffected brain parenchyma in all cases, as opposed to malignant hypercellular tumors which frequently exhibit a relatively restricted water diffusivity, particularly IDH-wild type gliomas $[11,13]$. Other sequences, such as perfusion-weighted imaging, have shown promise to discriminate glioma IDH status in a recent research [14]. In our cases, perfusion imaging was available in case 4 only. The rCBV was moderately increased (1.79) with respect to the normal appearing parenchyma, a non-specific value overlapping with other tumors [15]. 

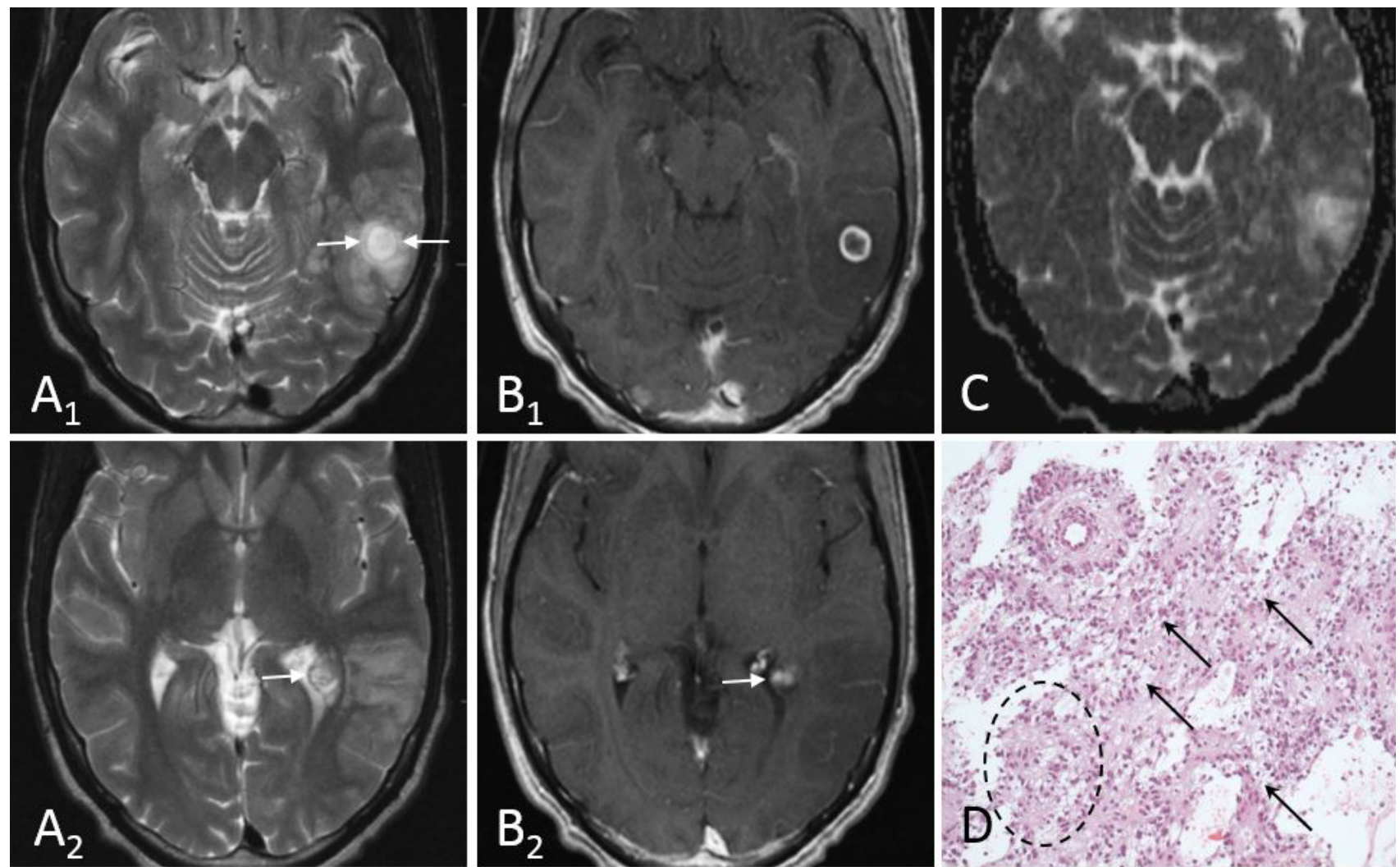

Fig. 5. Case 5. A case of multifocal and morphologically multifaceted aPA presentation. Axial T2-weighted image (A1) shows a cystic-like lesion with mild vasogenic edema in the left temporal lobe subcortical white matter (arrows), with a thin, T2-hypointense and intensely enhancing rim on T1-weighted postgadolinium images (B1). More caudally, (A2) shows a subependimal nodule abutting in the left ventricular atrium (arrow), with minimal vasogenic edema, and subtle core enhancement (arrow in B2). ADC map (C) demonstrates increased water diffusivity. Microscopically (D) this tumor exhibits a loose pattern with regular, round cells, with remarkable microcystic, pseudo-oligodendroglial changes (dashed circle), sometimes forming pseudorosette-like structures, with granular eosinophilic bodies (arrows) and rare mitoses. Ki-67 expression was approximately $10 \%$.

\subsection{Location}

With respect to the most frequent locations encountered in the pediatric population (the cerebellum and the optic pathways [1-3]) a relatively diverse distribution was noted in our aPA cases, involving both the infratentorial and supratentorial compartments. Four (57\%) were located infratentorially, one in the temporal lobe, and one in the lateral ventricle. Interestingly, case 5 presented with a multifocal pattern, a rare feature in PA that might reflect a dissemination along white matter tracts [16], and may be more frequently encountered in IDH-wild type than IDH-mutated glioma [11]. Three lesions (50\%) were located within the ventricular system and caused symptomatic hydrocephalus. Remarkably, one of these was centered in the mesencephalic aqueduct (case 1), which is, to the best of our knowledge, a location never described before for aPA. Therefore, PA could be considered in the differential diagnosis of intraventricular tumors more frequently encountered in adults in this location, including central neurocytoma, subependymoma, or chordoid glioma [10].

\subsection{Histopathology}

The majority of our adult cases showed a fibrillary matrix with Rosenthal fibers and eosinophilic granular bodies, typically encountered in pPA [17]. Interestingly, however, case 5 additionally exhibited some pseudo-oligodendroglial figures, a relatively unusual finding in pPA. Moreover, cases 2 and 6 well reflected the highly heterogeneous appearance seen on MRI, with both solid piloid textures, as well as cysticlike areas with necrosis and pleomorphic large cells. This large spectrum of morphology represents a potential challenge to the pathologist, and confirmatory molecular testing would be needed to definitely support diagnosis. Indeed, PAs may share many features with the glial component of ganglioglioma. Ganglioglioma is a well-differentiated glioneuronal tumor composed of dysplastic ganglion cells in combination with neoplastic glial cells [18]. In our opinion, the large ganglion-like cells seen in case 5 were likely related to regressive changes, rather than dysplastic alterations (see also Fig. 2G). These cells represented only a small part of the tumor, which was composed by a prominent neoplastic glial population. Additionally, we did not observe other histologic features typically identified in ganglioglioma, such as 

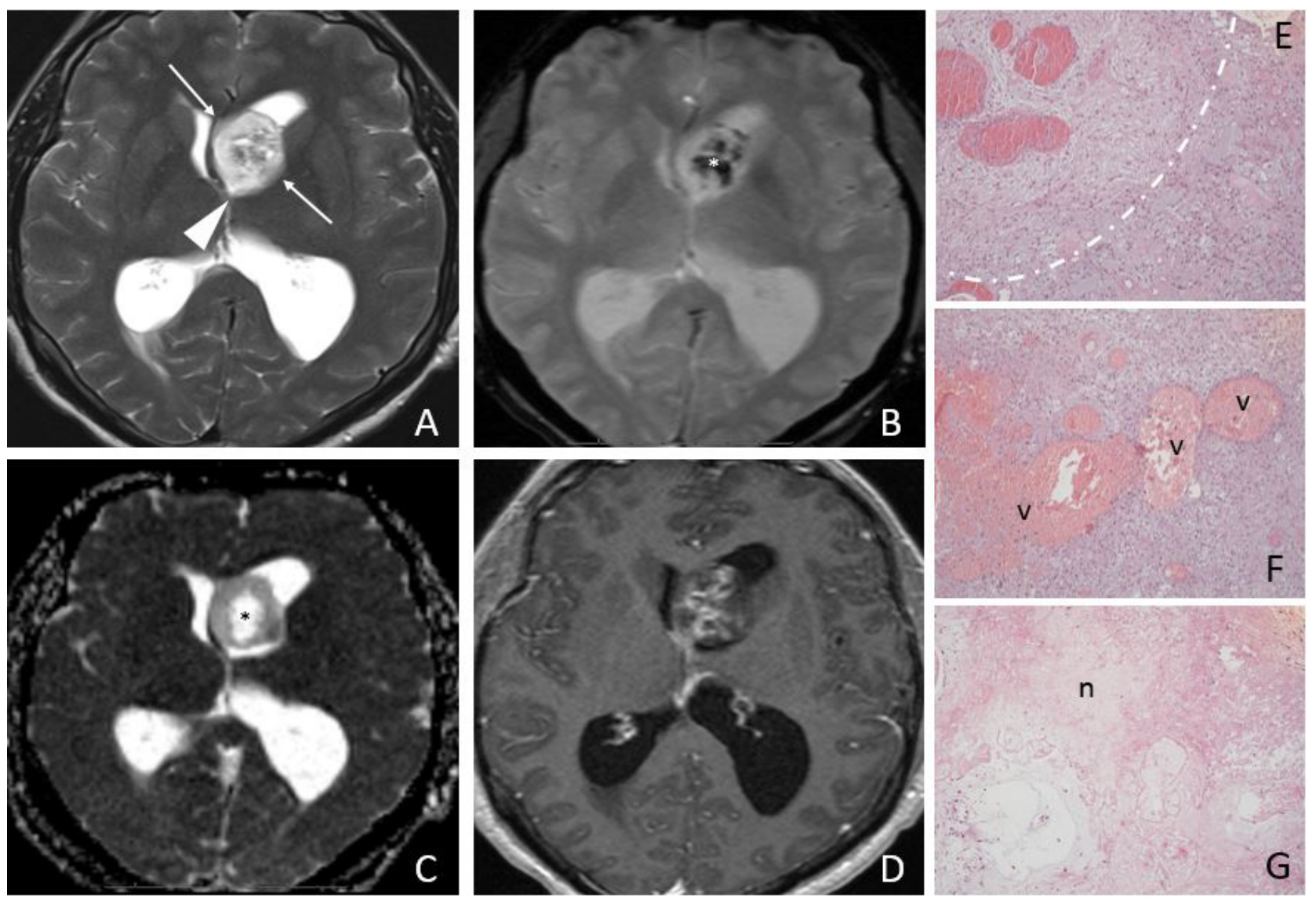

Fig. 6. Case 6. A case of supratentorial intraventricular aPA presenting with hydrocephalus, located in the left frontal ventricular horn and compressing the foramen of Monro. Axial T2-weighted (A) shows a hyperintense solid mass with heterogeneous core signal (arrows and arrowhead) and hemorrhagic deposits on T2*-weighted images (asterisk in C) indicative of necrosis. The corresponding T1-weighted image after gadolinium injection (D) shows marked inhomogeneous enhancement mostly at the level of the lesion core. Increased water diffusivity was demonstrated on the ADC map (asterisk in C). This tumor shows different histopathology patterns, varying from a relatively solid, biphasic arrangement (dashed line) alternating with compact and loose tissue textures containing mild to moderate cellularity (E), to other regions with ectatic hyalinized vessels ("v" in F). A necrotic area (n) with hemorrhage was also present (G).

calcifications, extensive lymphoid infiltrates in the perivascular spaces, tumor, brain parenchyma, and/or prominent capillary networks [18]. Importantly, while the BRAF V600E mutation represents the most common genetic alteration in ganglioglioma, it may not be employed per se as a diagnosismaking criterion because it is relatively frequent in PAs as well [19]. One limitation of our methodology was that we tested the BRAF gene exon 15 by direct sequencing only, without information about BRAF-KIAA1549 fusion. In a recent study, BRAF V600E mutation was detected in $8.9 \%$ of pediatric and $9.75 \%$ adult PAs, whereas $41.1 \%$ and $25.7 \%$ of pediatric and adult cases showed KIAA1549-BRAF fusions, respectively [20]. On the one end, testing for BRAFKIAA1549 fusion or BRAF V600E mutation may help to distinguish between distinct tumors with histologically similar appearances; on the other hand, these alterations are not present in the majority of PAs, and may not be used as a certainty criterion to make diagnosis. Another limitation is the lack of the H3 K27M mutation analysis. While pathologic features of the rare tumors harboring this alteration may mimic PAs, however H3 K27M mutated tumors usually show a high-grade histopathology appearance [21].

\section{Conclusions}

A variety of imaging presentation patterns characterized our aPA cases, corresponding to the heterogeneous and sometimes unusual tissue characteristics demonstrated by the histopathology results. PA rarely occurs in adulthood, but it might represent an MRI mimicker of higher grade tumors, particularly due to its variable morphology, MR signal and contrast enhancement features.

\section{Abbreviations}

PA, Pilocytic Astrocytoma; aPA, adult Pilocytic Astrocytoma; pPA, pediatric Pilocytic Astrocytoma; MRI, Magnetic Resonance Imaging; WHO, World Health Organization; CNS, central nervous system; TSE, Turbo-Spin-Echo; GRE, Gradient-Echo; rCBV, relative Cerebral Blood Volume; H\&E, hematoxylin and eosin. 


\section{Author contributions}

ADN performed cases collection and manuscript drafting. PS and LM provided histopathological data and images, and contributed to manuscript drafting. AC provided intellectual contribution and helped with manuscript review. EP sponsored, designed and performed final review of the work.

\section{Ethics approval and consent to participate}

The present work represents a report of six individual cases, and does not include patients' identifying information, therefore informed consent was not required.

\section{Acknowledgment}

The authors thank Vincent Tobe, Istituto Imaging della Svizzera Italiana, Svizzera, for help with manuscript editing.

\section{Funding}

This research received no external funding.

\section{Conflict of interest}

The authors declare no conflict of interest.

\section{References}

[1] Ostrom QT, Gittleman H, Truitt G, Boscia A, Kruchko C, Barnholtz-Sloan JS. CBTRUS Statistical Report: Primary Brain and other Central Nervous System Tumors Diagnosed in the United States in 2011-2015. Neuro-Oncology. 2018; 20: iv1-iv86.

[2] Bond KM, Hughes JD, Porter AL, Orina J, Fang S, Parney IF. Adult Pilocytic Astrocytoma: an Institutional Series and Systematic Literature Review for Extent of Resection and Recurrence. World Neurosurgery. 2018; 110: 276-283.

[3] Koeller KK, Rushing EJ. From the Archives of the AFIP: pilocytic astrocytoma: radiologic-pathologic correlation. RadioGraphics. 2004; 24: 1693-1708.

[4] Galgano MA, Padalino DJ, Fullmer J, Krishnamurthy S. Hemorrhagic Pilocytic Astrocytomas in Adults: A Case Report and Literature Review. Cureus. 2016; 8: e510.

[5] Fiechter M, Hewer E, Knecht U, Wiest R, Beck J, Raabe A, et al. Adult anaplastic pilocytic astrocytoma-a diagnostic challenge? A case series and literature review. Clinical Neurology and Neurosurgery. 2016; 147: 98-104.

[6] Soliman RK, Budai C, Mundada P, Aljohani B, Rushing EJ, Kollias SS. Suprasellar pilocytic astrocytoma in an adult with hemorrhage and leptomeningeal dissemination: case report and review of literature. Radiology Case Reports. 2016; 11: 411-418.

[7] Louis DN, Perry A, Reifenberger G, von Deimling A, Figarella-
Branger D, Cavenee WK, et al. The 2016 World Health Organization Classification of Tumors of the Central Nervous System: a summary. Acta Neuropathologica. 2016; 131: 803-820.

[8] Gaudino S, Martucci M, Russo R, Visconti E, Gangemi E, D'Argento F, et al. MR imaging of brain pilocytic astrocytoma: beyond the stereotype of benign astrocytoma. Child's Nervous System. 2017; 33: 35-54.

[9] Sato K, Rorke LB. Vascular bundles and wickerworks in childhood brain tumors. Pediatric Neuroscience. 1989; 15: 105-110.

[10] Osborn AG, Hedlund G, Salzman KL. Neoplasms, Cysts, and Tumor-Like Lesions. In Osborn's Brain (pp. 518-606). 2nd edn. Elsevier: Philadelphia. 2017.

[11] Villanueva-Meyer JE, Wood MD, Choi BS, Mabray MC, Butowski NA, Tihan T, et al. MRI Features and IDH Mutational Status of Grade II Diffuse Gliomas: Impact on Diagnosis and Prognosis. American Journal of Roentgenology. 2018; 210: 621-628.

[12] Chow D, Chang P, Weinberg BD, Bota DA, Grinband J, Filippi CG. Imaging Genetic Heterogeneity in Glioblastoma and other Glial Tumors: Review of Current Methods and Future Directions. American Journal of Roentgenology. 2018; 210: 30-38.

[13] Bull JG, Saunders DE, Clark CA. Discrimination of paediatric brain tumours using apparent diffusion coefficient histograms. European Radiology. 2012; 22: 447-457.

[14] Pasquini L, Napolitano A, Tagliente E, Dellepiane F, Lucignani M, Vidiri A, et al. Deep Learning Can Differentiate IDH-Mutant from IDH-Wild GBM. Journal of Personalized Medicine. 2021; 11: 290.

[15] Law M, Yang S, Wang H, Babb JS, Johnson G, Cha S, et al. Glioma grading: sensitivity, specificity, and predictive values of perfusion MR imaging and proton MR spectroscopic imaging compared with conventional MR imaging. American Journal of Neuroradiology. 2003; 24: 1989-1998.

[16] Faria AV, Azevedo GC, Zanardi VA, Ghizoni E, Queiroz LS. Dissemination patterns of pilocytic astrocytoma. Clinical Neurology and Neurosurgery. 2006; 108: 568-572.

[17] Matyja E, Grajkowska W, Stępień K, Naganska E. Heterogeneity of histopathological presentation of pilocytic astrocytoma - diagnostic pitfalls. a review. Folia Neuropathologica. 2016; 54: $197-$ 211.

[18] Blümcke I, Wiestler OD. Gangliogliomas: an intriguing tumor entity associated with focal epilepsies. Journal of Neuropathology and Experimental Neurology. 2002; 61: 575-584.

[19] Dougherty MJ, Santi M, Brose MS, Ma C, Resnick AC, Sievert $\mathrm{AJ}$, et al. Activating mutations in BRAF characterize a spectrum of pediatric low-grade gliomas. Neuro-Oncology. 2010; 12: 621-630.

[20] Kurani H, Gurav M, Shetty O, Chinnaswamy G, Moiyadi A, Gupta $\mathrm{T}$, et al. Pilocytic astrocytomas: BRAFV600E and BRAF fusion expression patterns in pediatric and adult age groups. Child's Nervous System. 2019; 35: 1525-1536.

[21] Daoud EV, Rajaram V, Cai C, Oberle RJ, Martin GR, Raisanen JM, et al. Adult Brainstem Gliomas with H3K27M Mutation: Radiology, Pathology, and Prognosis. Journal of Neuropathology \& Experimental Neurology. 2018; 77: 302-311. 